\title{
Effects of Ankaferd BloodStopper on bone healing in an ovariectomized osteoporotic rat model
}

\author{
ŞEREF EZIRGANLI ${ }^{1}$, HAKKI OĞUZ KAZANCIOĞLU ${ }^{2}$, AHMET HÜSEYIN ACAR ${ }^{2}$, \\ HAKAN ÖZDEMIR ${ }^{3}$, EMRE KUZU $^{4}$ and DENIZ ŞAHIN INAN ${ }^{5}$
}

\begin{abstract}
${ }^{1}$ Program of Dental Assisting, Vocational School of Health Services, Institute of Health Science, Nişantası University, Bayrampaşa, 34030 Istanbul; ${ }^{2}$ Department of Oral and Maxillofacial Surgery, Faculty of Dentistry, Bezmialem Vakif University, Fatih, 34093 Istanbul; ${ }^{3}$ Department of Periodontology, Faculty of Dentistry, Osmangazi University, 26480 Eskişehir; ${ }^{4}$ Dental Health Centre of Sivas, Rebublic of Turkey Ministry of Health, 58020 Sivas; ${ }^{5}$ Department of Histology and Embryology, Faculty of Medicine, Cumhuriyet University, 58140 Sivas, Turkey
\end{abstract}

Received May 23, 2016; Accepted October 28, 2016

DOI: $10.3892 /$ etm.2017.4166

\begin{abstract}
Bone defects resulting from oncological surgical resections, congenital facial anomalies, trauma or infection represent a significant and common clinical problem. The present study aimed to evaluate the effects of a commercially-available medicinal plant extract product, Ankaferd BloodStopper (ABS), on bone healing. The present study was performed on 24 female ovariectomized (OVX) rats. A defect on each rat calvarium was created using a trephine burr prior to the rats being divided into two groups. Defects were grafted with a gelatin sponge soaked with normal saline (control group) or plant extract (experimental group). Half of the animals were sacrificed after 2 weeks and the others after 4 weeks. In the control group, the defects were not filled with regenerated bone. By contrast, in the experimental group, all defect areas had an increased amount of regenerated bone and connective tissue. Osteoblastic activity appeared to be greater in the experimental group however, osteoclastic activity was observed to be higher in the control group. At 2 and 4 weeks, there was a significant difference in the amount of newly regenerated bone observed in the experimental group compared with the control group $(\mathrm{P}<0.05)$. Therefore, the results of the present study indicated that local ABS application had a positive effect on bone healing in the OVX rat model.
\end{abstract}

Correspondence to: Dr Hakki Oğuz Kazancioğlu, Department of Oral and Maxillofacial Surgery, Faculty of Dentistry, Bezmialem Vakif University, 1 Adnan Menderes Bulvarı, Fatih, 34093 Istanbul, Turkey

E-mail: dt_oguz@yahoo.com

Key words: Ankaferd BloodStopper, calvarial defect, osteoporosis, bone healing, osteoblast

\section{Introduction}

Bone defects resulting from facial trauma, congenital facial anomalies, oncological surgical resections or infection represent a common and significant clinical problem. Bone grafts such as autologous, allogenic or synthetic bone grafts, or prosthetic devices are used to reconstruct these defects (1). Autologous bone grafts are the gold standard for treating bone defects, however, bone supply is limited and a second surgical site is required (2). Therefore, allogeneic bone grafts from donors or cadavers are generally used as an alternative graft material to autologous bone grafts. However, their osteoinductive capability remains controversial and there is an increased risk of infections, including hepatitis and HIV (1). Similarly, prosthetic devices are plagued by the potential risk of infection and extrusion (3). Due to these limitations, a number of experimental studies have aimed to improve the healing of calvarial bone defects $(1,4-7)$. Studies investigating various antioxidants including propolis (8), Nigella sativa (9), Ginkgo biloba (10) and Ankaferd BloodStopper (ABS) (11) have been performed to assess their effects on bone healing. ABS is a plant extract product composed of Glycyrrhiza glabra, Vitis vinifera, Thymus vulgaris, Alpinia officinarum and dried root of Urtica dioica, approved for use in the management of external haemorrhage and surgical bleeding by the Turkish Ministry of Health (Report No: B.10.1.RSH.0.08.01.St./17422; Ankara, Turkey).

The formation of an encapsulated protein network representing focal points for vital erythrocyte aggregation, is the basic mechanism of haemostasis (12). Osteoporosis is a chronic skeletal disease defined by a pathological decrease in the amount of bone and characterized by deterioration and reduction of the micro-architecture of bone tissue, leading to fractures that occur following minimal trauma $(13,14)$. Osteoporosis can be divided into two groups, primary (postmenopausal, geriatric) and secondary, caused by etiological factors such as oestrogen deficiency, which is often detected in females with postmenopausal osteoporosis $(15,16)$. The ovariectomized (OVX) rat is an appropriate model for studies investigating human postmenopausal osteoporosis due to 
a number of similarities in the pathophysiological mechanisms (15). Postmenopausal bone loss and ovariectomy-induced bone loss in the rat share a number of similar characteristics, including an initial rapid phase of bone loss followed by a much slower phase, an increased rate of bone turnover with resorption exceeding formation, decreased intestinal absorption of calcium and a greater loss of cancellous bone compared with cortical bone $(15,16)$. These similarities suggest that the OVX rat model is suitable for the study of problems associated with postmenopausal bone loss (16). Therefore, the present study aimed to evaluate the potential of ABS to support bone regeneration in calvarial defects in an OVX osteoporotic rat model.

\section{Materials and methods}

Animal models. A total of 24 female Wistar rats were used, with a mean body weight of $250 \pm 18 \mathrm{~g}$, aged 3 months. They were obtained from the Bezmialem Vakif University Laboratory Animal Center, Istanbul. The animals were kept in individual plastic cages in an experimental animal room at $22^{\circ} \mathrm{C}$ with a 12-h light/dark cycle. The animals of the study and control groups were fed a standard laboratory pellet diet and drinking water was available ad libitum throughout the experiment. The experimental procedures of the present study were approved by the Animal Ethics Committee of Cumhuriyet University School of Medicine (B.30.2.CUM.0.01.00.00-50/110; Sivas, Turkey). The present study was conducted according to the principles of the Helsinki Declaration related to the protection of laboratory test animals.

Ovariectomy procedure. Surgery was completed under sterile conditions. Rats were anesthetized by intramuscular injection of $5 \mathrm{mg} / \mathrm{kg}$ Rompuns ${ }^{\mathrm{TM}}$ (Bayer AG, Leverkusen, Germany) and $40 \mathrm{mg} / \mathrm{kg} \mathrm{Ketalar}{ }^{\circledR}$ (Eczacıbaşı, Holding, Istanbul, Turkey). The abdominal skin of each rat was cleaned with a povidone-iodine solution. A $2-\mathrm{cm}$ midline dorsal skin incision was made and the connective tissue between the skin and muscular layer of the abdominal wall was dissected with a dorsal approach. The ovaries were excised bilaterally following ligation of the uterine horn. The muscle layers and skin were then sutured with coated Vicryl ${ }^{\circledR}$ 4-0 polyglactin 910 (Ethicon; Johnson \& Johnson, Somerville, NJ, USA). To prevent postoperative infection, an intramuscular injection of $30 \mathrm{mg} / \mathrm{kg}$ ceftriaxon (Roche Diagnostics, Basel, Switzerland) and $4 \mathrm{mg} / \mathrm{kg}$ carprofen (Pfizer, Inc., New York, NY, USA) was administered to the animals every $24 \mathrm{~h}$ for 3 days starting immediately following the surgery. The oestrous cycle was monitored to confirm the success of the ovariectomy and a vaginal smear was examined for 4-5 days throughout the oestrous cycle in all the rats.

Experimental design. The study was started 12 weeks following OVX surgery. The calvarium defects were formed as described (4-6) and the animals were divided into two groups of 12 rats: A control group and an ABS (Ankaferd Health Products Ltd., Istanbul, Turkey) experimental group. In the control group, the defect was grafted at a $2 \mathrm{~mm}$ thickness and a $5 \mathrm{~mm}$ diameter, with a circular gelatin sponge, mixed with $1.5 \mathrm{mg}$ saline solution. Defects in the ABS group were grafted at a $2 \mathrm{~mm}$ thickness and a $5 \mathrm{~mm}$ diameter, with a

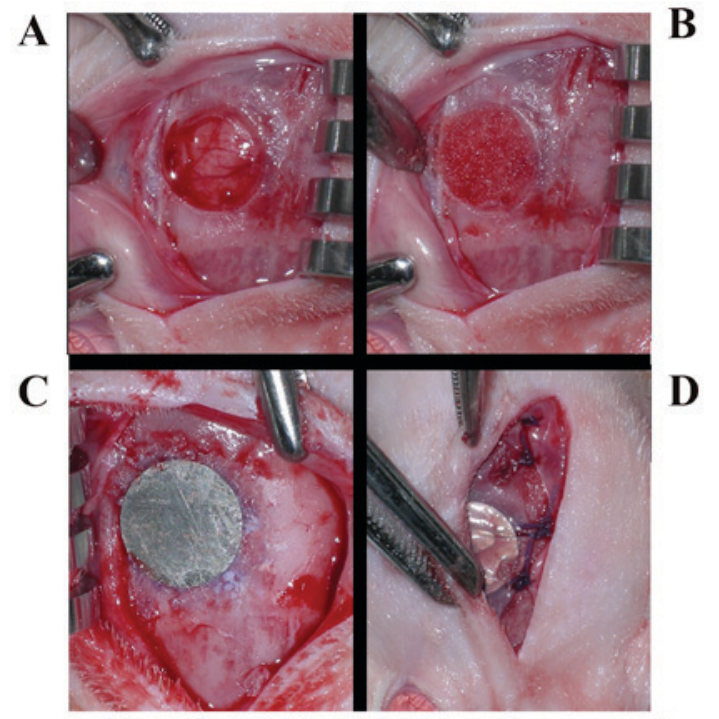

Figure 1. Surgical procedure in control and experimental mice. (A) A defect on the calvarium was created with a diameter trephine burr. (B) In the control group the defect area was grafted with gelatin sponge mixed with saline solution and the experimental group was grafted with gelatin sponge mixed with Ankaferd BloodStopper. (C) Titanium disc fixed to the calvarial bone completely over the defect. (D) Periosteum and then the skin were sutured.

circular gelatin sponge, mixed with $1.5 \mathrm{mg}$ ABS. In all control and experimental groups, ABS and saline were administered locally, once during the surgery. All defects were covered with a thin polished titanium disc, which extended beyond each border of the defect. Half of the animals in each group were sacrificed with an overdose of $200 \mathrm{mg} / \mathrm{kg}$ intravenous (IV) pentobarbital (Pentothal, Abbott Laboratories, Abbott Park, IL, USA) after 2 weeks, and the other half after 4 weeks. Rats received the same treatment protocol as rats in the same groups that were sacrificed at different times.

Surgical procedure. Following general anesthesia [intramuscular injection of $3 \mathrm{mg} / \mathrm{kg}$ xylazine hydrochloride (Rompuns, Bayer, Leverkusen, Germany) and $35 \mathrm{mg} / \mathrm{kg}$ ketamine hydrochloride (10\% Ketasol ${ }^{\circledR}$, Richter Pharma AG, Wels, Austria)], all rat calvariums were shaved and the cutaneous surfaces were disinfected with povidone iodine solution prior to surgery. In order to raise the skin of the calvarium, a skin incision $\sim 2.5 \mathrm{~cm}$ long was made over the linea media. A small sharp periosteal elevator was used to raise the cutaneous flap laterally and the periosteum was incised and lifted to expose the calvarium. Following exposure of the calvarium, a defect on the right calvarium of each rat was created with a standard 5-mm diameter trephine bur. Defects in the control groups were grafted with a gelatin sponge mixed with normal saline and the experimental groups were grafted with a gelatin sponge soaked in ABS. In all animals, titanium discs were fixed to the calvarial bone completely over the defects, using Histoacryl ${ }^{\circledR}$ (B. Braun Melsungen AG, Melsungen, Germany; Fig. 1). The periosteum and the skin were carefully sutured with Vicryl ${ }^{\circledR}$ 4-0 polyglactin 910 sutures (Vicryl; Ethicon, Somerville, NJ, USA). To prevent infection, the aforementioned doses of ceftriaxon antibiotic and carprofen analgesic were injected intra-muscularly into the animals every $24 \mathrm{~h}$ for 3 days starting immediately following the surgery. 
Specimen preparation. The area of the original surgical defect and the surrounding tissues were removed en bloc from the calvarium, following sacrifice. The blocks were fixed in $10 \%$ formalin at room temperature for 24-72 h. Formalin was rinsed from the specimens with running water and they were decalcified in $10 \%$ formic acid for 7 days. Each specimen was then bisected longitudinally into two blocks in a sagittal direction and embedded in paraffin blocks. For each animal, 2 serial sections $\sim 6-\mu \mathrm{m}$ thick were cut in a longitudinal direction beginning at the centre of the original surgical defect. The sections were stained with hematoxylin-eosin for histomorphometric analysis under an Eclipse E 600 light microscope (Nikon Corporation, Tokyo, Japan).

Histological and histomorphometric analysis. The same histologist, who was blinded to the identity of the samples, performed histological and histomorphometric analyses. The presence of inflammatory infiltrate, connective tissue, resorption and bone regeneration was evaluated and the number of osteoblasts and osteoclasts in the defect area were counted. A digital camera connected to a light microscope with an original magnification of $x 40$ captured images of the histological sections in all groups and saved them on a computer. For histomorphometric analysis, Clemex Vision-Lite 5.0 software (Clemex Technologies, Inc., Quebec, Canada) was used and the area of newly formed bone within the original surgical defect was calculated using an automated image analysis system (NIS Elements version 4.0, Nikon Corporation, Tokyo, Japan) to generate computer-assisted histomorphometic measurements.

Statistical analysis. All statistical analyses were performed using SPSS, version 20.0 (IBM SPSS, Armonk, NY, USA). The data were subjected to statistical analysis with the Mann-Whitney U test followed by the Kruskal-Wallis test for intergroup comparison and the Wilcoxon signed-rank test for comparisons between the 2 - and 4 -week groups. $\mathrm{P}<0.05$ was considered to represent a statistically significant difference.

\section{Results}

No damage to the dura mater was observed in any of the specimens. No inflammatory processes were noted in any of the groups. Connective tissue with collagen fibres parallel to the defect surface and a moderate number of fibroblasts were detected in all groups.

Histological analysis. In the control groups, similar histological results were observed in the 2- and 4-week groups; no defects were completely filled with regenerated bone. No ossification was identified in the defect area of the 2-week control group (Fig. 2A). In the 4-week control group, loose and thin connective tissues in the defect area and areas of mineralized bone were identified. Ossification was minimal, originating from the defect margins (Fig. 2B).

In the 2-week experimental group, all defect areas were mostly filled with regenerated bone and connective tissue. The presence of dense fibroblast connective tissue, dense ossification, osteoblast and osteoclast cells in the defect area were notable (Fig. 3). In the 4-week experimental group, bone

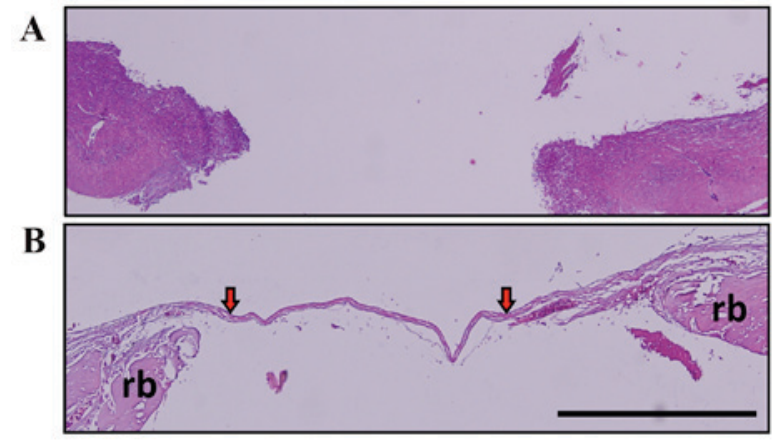

Figure 2. Histological assessment. (A) No defect was completely filled in the 2-week control group; the histological image is observed at original magnification (hematoxylin and eosin, x40) (B) Loose and thin connective tissues in the defect areas and regenerated bone areas were observed in the 4-week control group. Red arrows indicate the presence of the thin connective tissue in the defect area. Minimal ossification was detected originating from the defect margins (hematoxylin and eosin, x100). Scale bar, $0.5 \mathrm{~mm}$. $\mathrm{Rb}$, regenerated bone.

A

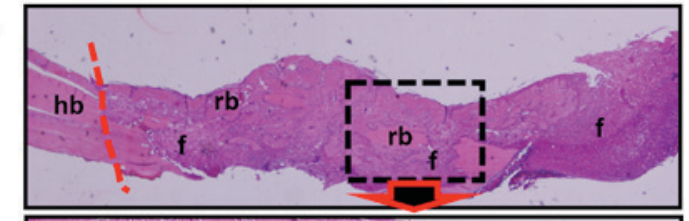

B

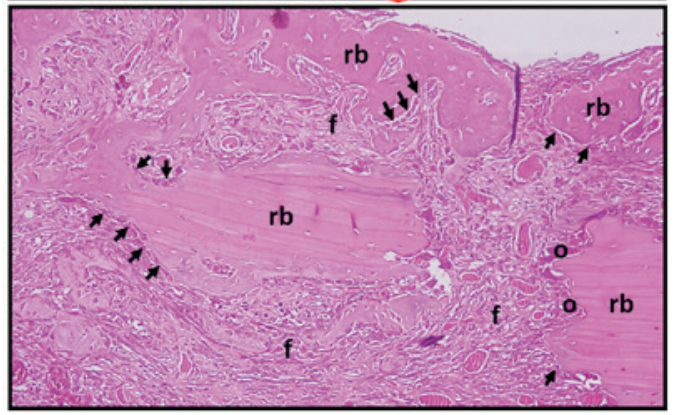

Figure 3. Histological assessment for the 2-week experimental and control groups. (A) Regenerated bone areas with fibrocellular connective tissue and host bone area boundaries are observed (hematoxylin and eosin, x100). (B) The presence of dense fibrocellular connective tissue, dense ossification, osteoblasts, and osteoclast cells are indicated (hematoxylin and eosin, x400). Arrow, osteoblast; o, osteoclast; f, fibrocellular connective tissue; hb, host bone; rb, regenerated bone; line (---), border of host bone tissue and regenerated tissues; ABS, Ankaferd BloodStopper.

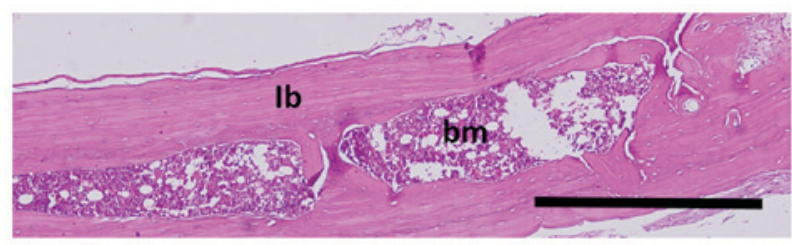

Figure 4. Histological assessment for the 4-week experimental and control groups. Bone growth was complete, formed of $\mathrm{lb}$ and bm (hematoxylin and eosin, x200). Scale bar, $0.5 \mathrm{~mm}$. lb, lamellar bone; bm, bone marrow.

growth was observed to be complete, formed of lamellar bone and bone marrow (Fig. 4).

Histomorphometric analysis. In a comparison of all groups, osteoblastic activity was observed to be greater in the 


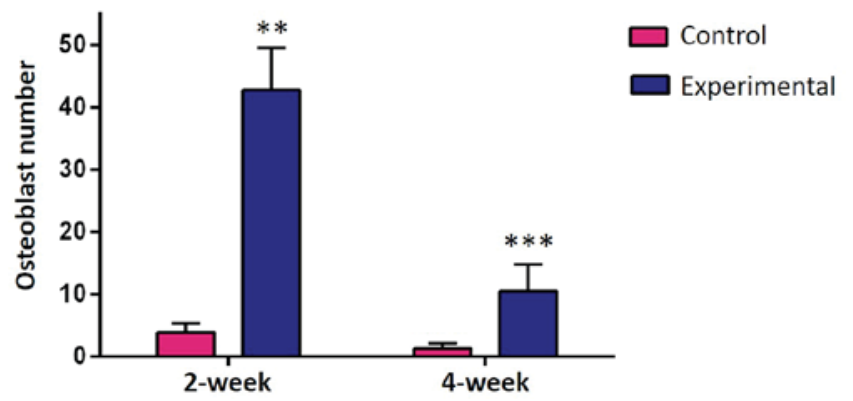

Figure 5. Histomorphometric analysis of osteoblastic activity. Experimental groups exhibited increased osteoblastic activity compared with control groups. ${ }^{* *} \mathrm{P}<0.01,{ }^{* * *} \mathrm{P}<0.01$ vs. control.

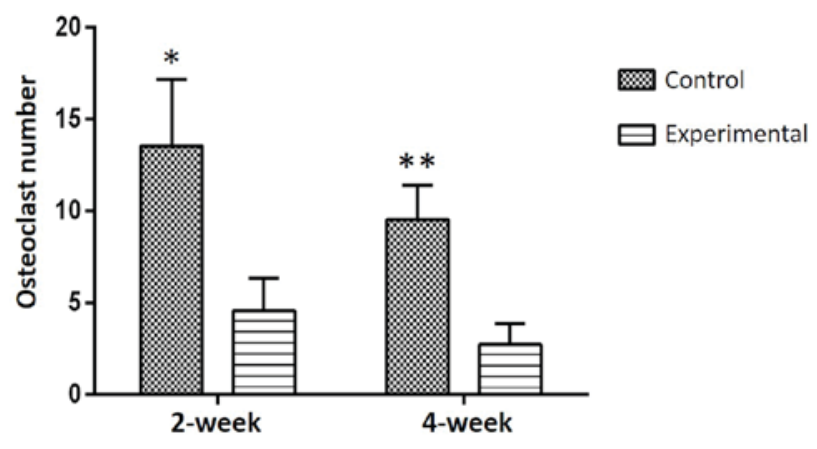

Figure 6. Histomorphometric analysis of osteoclastic activity was higher in the control groups compared with the experimental groups ${ }^{*} \mathrm{P}<0.05,{ }^{* *} \mathrm{P}<0.05$ vs. experimental groups.

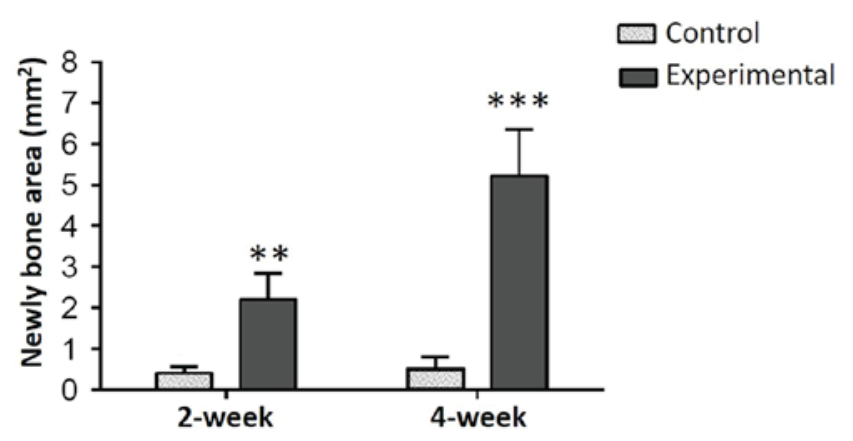

Figure 7. A comparison of 2 and 4-week control and experimental groups. A statistically significant difference was determined between the control and experimental groups at 2 and 4 weeks with regard to histomorphometric analysis of regenerated bone. ${ }^{* *} \mathrm{P}<0.05,{ }^{* * * *} \mathrm{P}<0.05$ vs. control.

experimental groups than in the control groups ( $\mathrm{P}<0.01$; Fig. 5) and osteoclastic activity was observed to be greater in the control groups ( $\mathrm{P}<0.05$; Fig. 6$)$ at weeks 2 and 4 . In all groups, more ossification was evident in the 4-week groups compared with the 2-week groups. Among the experimental groups, no significant difference was identified between the number of osteoclast cells in the 2-week and 4-week groups. The amount of regenerated bone in the defect area was measured as $\mathrm{mm}^{2}$ during the histomorphometric assessment. Comparisons of all the groups are presented in Fig. 7. A statistically significant difference was determined between the control and experimental groups with respect to regenerated bone at both 2 and 4 weeks $(\mathrm{P}<0.05$; Fig. 7$)$.

\section{Discussion}

Animal models serve a critical role in bone-related studies, particularly in the assessment of the biological characteristics of bone metabolism, in physiological and pathological conditions. Additionally, experimental animals have been used extensively to model human pathological states, including osteoporosis (17).

In the present study, a rat calvarial defect model was used to evaluate bone regeneration. This model is considered comparatively locatable, simple and reproducible as in this defect, spontaneous healing does not occur. Furthermore, the model was observed to be effective at evaluating the potential for bone regeneration. Finally, this model has some compressive force similar to intraoral conditions (4). In experimental animal studies, the calvarial defect model is considered to be the most exclusive experimental model of bone formation due to poor blood supply and the membranous structure, which preclude any spontaneous healing (5).

Osteoporosis is associated with a decreased regenerative capacity of bone, which may account for the clinical impairment observed with bone-regenerative approaches in osteoporotic models $(15,16)$. The OVX rat model is commonly used in studies investigating the pathophysiology, diagnosis and therapy of osteoporosis and has been validated as a clinically relevant model of human postmenopausal bone loss (17). Oestrogen deficiency results in increased bone turnover and imbalance in favour of resorption, causing osteoporosis. Luize et al (18) demonstrated that oestrogen depletion, caused by ovariectomy, hinders bone healing in rats. Thus, the impact of osteoporosis on bone graft healing and its long-term outcome requires attention (14). A number of studies using an OVX rat model have been recorded in literature $(15,18)$. These studies were completed between 4-12 weeks following OVX surgery however, in the present study, bilateral ovariectomy of the OVX rat model was performed and the study was started 12 weeks after OVX surgery.

Bone regeneration is a complex biological process with three key components: A morphogenetic signal, a convenient carrier matrix that acts as a scaffold for bone regeneration and responding host cells that are able to differentiate into bone cells $(15,18)$. Bioactive factors including bone morphogenic proteins (BMP), transforming growth factor-beta (TGF- $\beta$ ) fibroblast growth factor-2 and platelet-derived growth factor serve a role in bone healing, either alone or in combination $(19,20)$. Maeda et al (21) assessed whether bone anabolic factors other than BMP-2 are induced by the treatment of osteoblasts with statins. It was determined that statins including atorvastatin, simvastatin and cerivastatin markedly enhanced the expression of vascular endothelial growth factor in three osteoblastic cell lines. Nyan et al (22) recognized that the recruitment and proliferation of osteoprogenitor cells were critical steps in the early stage of bone healing. These steps were enhanced by TGF- $\beta$ and BMP-2, which were in turn, stimulated by simvastatin. In addition, Ezirganli et al (23) demonstrated that local application of simvastatin enhanced bone healing in the calvarial defect model.

ABS is a commercially-available plant extract product, which has been used as a haemostatic agent in Anatolia, Turkey. Its usage has been approved in Turkey for use in 
the management of dental surgery bleeding and external haemorrhage (12). The product contains a standardized mixture of five plants and although it has been used historically as a haemostatic agent, its mechanism of action remains unknown (24). The five plants included are: Dried leaf of Glycyrrhiza glabra, Vitis vinifera, Thymus vulgaris, Alpinia officinarum and the dried root of Urtica dioica. Each of these plants has an effect on the endothelium, cellular proliferation, blood cells, angiogenesis, cell mediators or vascular dynamics. It has been demonstrated that Glycyrrhiza glabra has angiogenic, antithrombin, anti-inflammatory, antiatherosclerotic, antiplatelet, antioxidant and antitumor activities (24). Thymus vulgaris exhibits an anti-oxidant effect, Alpinia officinarum is effective against rheumatism, bad breath, bronchial catarrh and ulcers, Vitis vinifera has antioxidative, anti-inflammatory and antimicrobial activities, and Urtica dioica has acute diuretic, hypotensive and cardiovascular effects $(12,24)$.

A number of studies have focused on bone healing in the calvarial defect $(4,23)$; however, to the best of our knowledge, this is the first study investigating the effect of ABS on bone healing. The present study used a handmade titanium disc to completely close and protect the calvarial defect area, unlike other studies $(5,12)$. Although there are numerous studies $(12,24,25)$ concerned with the hemostatic effects of ABS, studies associated with the effects of ABS on bone healing are rare and there have been no studies, to the best of our knowledge, using ABS in the OVX rat model. There has been one study observing the effects of ABS on bone healing. Işler et al (11) demonstrated that in early bone healing, ABS decreased inflammation and necrosis, and increased new bone formation. Furthermore, no foreign body reaction to ABS was detected (11). Similarly in the present study, the local application of ABS was observed to have significantly increased bone regeneration in the calvarial defect.

In conclusion, the present study presented certain limitations, including the fact that it was an animal study with a small sample size. However, the local application of ABS had a positive effect on bone healing in the OVX rat model. Further experimental and clinical studies focused on ABS may be required for determining the therapeutic dose of ABS.

\section{References}

1. Terella A, Mariner P, Brown N, Anseth K and Streubel SO: Repair of a calvarial defect with biofactor and stem cell-embedded polyethylene glycol scaffold. Arch Facial Plast Surg 12: 166-171, 2010.

2. Ezirganlı Ş, Polat S, Barış E, Tatar İ and Çelik HH: Comparative investigation of the effects of different materials used with a titanium barrier on new bone formation. Clin Oral Implants Res 24: 312-319, 2013.

3. Rubin JP and Yaremchuk MJ: Complications and toxicities of implantable biomaterials used in facial reconstructive and aesthetic surgery: A comprehensive review of the literature. Plast Reconstr Surg 100: 1336-1353, 1997.

4. Choi JY, Jung UW, Kim CS, Eom TK, Kang EJ, Cho KS, Kim CK and Choi SH: The effects of newly formed synthetic peptide on bone regeneration in rat calvarial defects. J Periodontal Implant Sci 40: 11-18, 2010.

5. Toker H, Ozdemir H, Ozer H and Eren K: Alendronate enhances osseous healing in a rat calvarial defect model. Arch Oral Biol 57: 1545-1550, 2012.
6. Acar AH, Yolcu Ü, Gül M, Keleş A, Erdem NF and Altundag Kahraman S: Micro-computed tomography and histomorphometric analysis of the effects of platelet-rich fibrin on bone regeneration in the rabbit calvarium. Arch Oral Biol 60: 606-614, 2015.

7. Acar AH, Yolcu Ü, Altındiş S, Gül M, Alan H and Malkoç S: Bone regeneration by low-level laser therapy and low-intensity pulsed ultrasound therapy in the rabbit calvarium. Arch Oral Biol 61: 60-65, 2016

8. Guney A, Karaman I, Oner M and Yerer MB: Effects of propolis on fracture healing: An experimental study. Phytother Res 25: 1648-1652, 2011.

9. Kirui PK, Cameron J, Benghuzzi HA, Tucci M, Patel R, Adah F and Russell G: Effects of sustained delivery of thymoqiunone on bone healing of male rats. Biomed Sci Instrum 40: 111-116, 2004

10. Duygulu F, Yakan B, Karaoglu S, Kutlubay R, Karahan OI and Ozturk A: The effect of zymosan and the protective effect of various antioxidants on fracture healing in rats. Arch Orthop Trauma Surg 127: 493-501, 2007.

11. Işler SC, Demircan S, Cakarer S, Cebi Z, Keskin C, Soluk M and Yüzbaşioğlu E: Effects of folk medicinal plant extract Ankaferd Blood Stopper on early bone healing. J Appl Oral Sci 18: 409-414, 2010.

12. Kazancıoğlu HO, Cakır O, Ak G and Zülfikar B: The effectiveness of a new hemostatic agent (ankaferd blood stopper) for the control of bleeding following tooth extraction in Hemophilia: A controlled clinical trial. Turk J Haematol 30: 19-24, 2013.

13. Tamimi FM, Torres J, Tresguerres I, Clemente C, LópezCabarcos E and Blanco LJ: Bone augmentation in rabbit calvariae: Comparative study between Bio-Oss and a novel beta-TCP/DCPD granulate. J Clin Periodontol 33: 922-928, 2006.

14. Akimoto K, Becker W, Donath K, Becker BE and Sanchez R: Formation of bone around titanium implants placed into zero wall defects: Pilot project using reinforced e-PTFE membrane and autogenous bone grafts. Clin Implant Dent Relat Res 1: 98-104, 1999.

15. Comelekoglu U, Bagis S, Yalin S, Ogenler O, Yildiz A, Sahin NO, Oguz I and Hatungil R: Biomechanical evaluation in osteoporosis: Ovariectomized rat model. Clin Rheumatol 26: 380-384, 2007.

16. Kalu DN: The ovariectomized rat model of postmenopausal bone loss. Bone Miner 15: 175-191, 1991.

17. Herron S, Thordarson DB, Winet H, Luk A and Bao JY: Ingrowth of bone into absorbable bone cement: An in vivo microscopic evaluation. Am J Orthop (Belle Mead NJ) 32: 581-584, 2003.

18. Luize DS, Bosco AF, Bonfante S and de Almeida JM: Influence of ovariectomy on healing of autogenous bone block grafts in the mandible: A histomorphometric study in an aged rat model. Int J Oral Maxillofac Implants 23: 207-214, 2008.

19. Thorwarth M, Schultze-Mosgau S, Wehrhan F, Kessler P, Srour S, Wiltfang J and Andrea Schlegel K: Bioactivation of an anorganic bone matrix by P-15 peptide for the promotion of early bone formation. Biomaterials 26: 5648-5657, 2005.

20. Merkx MA, Maltha JC, Freihofer HP and Kuijpers-Jagtman AM: Incorporation of particulated bone implants in the facial skeleton. Biomaterials 20: 2029-2035, 1999.

21. Maeda T, Kawane T and Horiuchi N: Statins augment vascular endothelial grow th factor expression in osteoblastic cells via inhibition of protein prenylation. Endocrinology 144: 681-692, 2003.

22. Nyan M, Miyahara T, Noritake K, Hao J, Rodriguez R, Kuroda S and Kasugai S: Molecular and tissue responses in the healing of rat calvarial defects after local application of simvastatin combined with alpha tricalcium phosphate. J Biomed Mater Res B Appl Biomater 93: 65-73, 2010.

23. Ezirganli S, Kazancioğlu HO, Mihmanli A, Aydin MS, Sharifov R and Alkan A: The effect of local simvastatin application on critical size defects in the diabetic rats. Clin Oral Implants Res 25: 969-976, 2014.

24. Goker H, Haznedaroglu IC, Ercetin S, Kirazli S, Akman U, Ozturk Y and Firat HC: Haemostatic actions of the folkloric medicinal plant extract Ankaferd Blood Stopper. J Int Med Res 36: 163-170, 2008.

25. Leblebisatan G, Bay A, Karakus SC, Kekilli M and Haznedaroglu IC: Topical Ankaferd hemostat application for the management of oral cavity bleedings in children with hemorrhagic diathesis. Blood Coagul Fibrinolysis 23: 494-497, 2012. 